\title{
Qualidade de vida em sobreviventes de acidente vascular cerebral - instrumentos de avaliação e seus resultados
}

Quality of life in stroke survivors - assessment

instruments and their outcomes

Juliana Ferreira Mota', Rodrigo Nicolato ${ }^{1}$

\section{RESUMO}

Objetivos: Os objetivos deste estudo foram identificar os instrumentos genéricos e específicos utilizados na avaliação da qualidade de vida (QV) e os seus resultados em sobreviventes de acidente vascular cerebral (AVC). Métodos: Realizou-se revisão da literatura dos últimos dez anos, com população acima de 18 anos, nos bancos de dados MedLine e Lilacs, cujas publicações utilizassem instrumentos padronizados e validados no país de origem. Combinaram-se os descritores quality of life, cerebrovascular accident, stroke, QV e acidente cerebrovascular. Resultados: Consideraram-se relevantes 96 estudos e 31 entram neste trabalho, de acordo com os critérios de inclusão. Foram encontrados cinco tipos diferentes de instrumentos genéricos/perfil, nove genérico/utility e dois específicos. O mais freqüente foi o SF-36, em 45,2\% dos estudos. Observou-se que a baixa QV relacionou-se, principal-

\section{Palavras-chave}

Qualidade de vida, acidente cerebrovascular, acidente vascular cerebral, instrumentos.

\section{Keywords}

Quality of life, cerebrovascular accident, stroke; instruments. mente, ao déficit da função física, à presença de depressão ou de seus sintomas, ser do sexo feminino e ser mais idoso. De modo geral, os sujeitos no pós-AVC possuíam pior QV do que aqueles que não sofreram o evento. Conclusão: Foram encontrados 16 instrumentos para avaliação da QV. A baixa QV foi prevalente nos sobreviventes pós-AVC e se correlacionou com a função física, a depressão, o sexo e a idade.

\section{ABSTRACT}

Objective: The purpose of this study is to identify generical and specific instruments used for valueing quality of life (QOL) and their outcomes in stroke survivors. Methods: Review of literature of last 10 years, with people above 18 years old, in MedLine and Lilacs database. The instruments used on the studies were validated for the their countries. 96 articles have been considered relevant and 31 were in accordance with inclusion criteria. Five kind of generic/profile, nine generic/ utility and two specific instruments were found. The more frequent was SF-36, on the $45,2 \%$ of the studies. It has been observed that poverty in quality of life was related to defective the physical function, to the presence of depression or its symptoms, to the feminine gender and to being older. In general, survivors of cerebrovascular accident had worse QOL than the ones who did not suffer it. Conclusion: It has been found 16 instruments for QOL' assessment. The poverty of QOL was frequent on stroke survivors and was related to physical function, depression, sex and age.

Instituto de Previdência dos Servidores do Estado de Minas Gerais (IPSEMG)

Recebido em

$8 / 4 / 2008$

Aprovado em

$27 / 6 / 2008$

Endereço para correspondência: Juliana Ferreira Mota

Rua Adolfo Ciolete, 760 - Barreiro - 30626-440 - Belo Horizonte, MG

E-mail: julianaferreiramota@yahoo.com.br 


\section{INTRODUÇÃO}

A qualidade de vida (QV) foi definida pelo grupo de qualidade de vida da Organização Mundial de Saúde (OMS) como "a percepção do indivíduo de sua posição na vida, no contexto da cultura e sistemas de valores nos quais ele vive e em relação aos seus objetivos, expectativas, padrões e preocupações"1. Muitos estudos têm feito o intercâmbio entre QV e os termos QV relacionados à saúde (QVRS), estado de saúde e bem-estar subjetivo ${ }^{2-4}$. No entanto, a definição desses termos não é clara. Gill e Feinsten ${ }^{5}$ examinaram 75 artigos descrevendo o uso de instrumentos de QV. Destes, somente 15\% definiram o que era QV ou justificaram a escolha de determinado instrumento.

Um instrumento de avaliação da QV não deve se limitar a medir a presença ou a gravidade de determinada doença. De maneira geral, a percepção individual tem sido avaliada em grandes domínios ou dimensões da vida. Em termos práticos, os domínios são conjuntos de questões agrupadas nos instrumentos de avaliação e que se referem a determinada área do comportamento ou da condição humana, por exemplo, os domínios psicológico, físico, social e ambiental. Foi particularmente na década de 1960 que o construto QV passou a ser entendido como subjetivo e multidimensional'.

Não existe um único instrumento capaz de avaliar todas as situações de doença e saúde. Assim, a escolha do instrumento deve se associar ao objetivo do estudo e também de sua disponibilidade no idioma e no contexto cultural no qual possa ser empregado.

A literatura científica classifica os instrumentos de avaliação de QV como genéricos e específicos?. Os instrumentos genéricos têm como vantagens a possibilidade de avaliação simultânea de várias áreas ou domínios, de poderem ser usados em qualquer população e o fato de permitirem comparações entre pacientes com diferentes patologias. A grande desvantagem é não demonstrar alterações em aspectos específicos. Eles ainda podem ser subdivididos em perfil de saúde e medidas de utility. O primeiro proporciona ampla avaliação de diferentes aspectos referentes à QV, e os segundos são derivados de teorias econômicas e de decisão, refletindo a preferência do indivíduo pelo estado de saúde, pelo tratamento ou pela intervenção $0^{6,7}$. Estes últimos permitem a estruturação de árvores de decisão, assim como o cálculo de anos de vida ajustados pela qualidade. Dessa forma, a preferência do indivíduo é sumarizada dentro da escala que varia de 0 (zero) a 1 (um), sendo 0 igual a morte e 1 igual a saúde perfeita ${ }^{6}$.

Os instrumentos específicos são destinados a determinadas doenças, populações e funções. Eles avaliam, de maneira individual e específica, aspectos da QV. Sua principal vantagem é o seu potencial de ser sensível às alterações e aos aspectos particulares. Sua desvantagem é não permitir comparações entre populações distintas, além de ser restrito aos domínios de relevância do aspecto a ser avaliado.

Os aprimoramentos científico e tecnológico têm proporcionado aumento da sobrevida dos pacientes. Estes, então, passaram a viver mais tempo com os problemas e as morbidades decorrentes de suas doenças crônicas ${ }^{8}$. A American Heart Association estima que haja nos Estados Unidos cerca de quatro milhões de sobreviventes a acidentes vasculares cerebrais (AVC) e que ocorrem aproximadamente 600 mil casos novos por ano ${ }^{4}$. O número de casos fatais tem diminuído. Cerca de $85 \%$ dos indivíduos sobrevivem ao AVC e vivem com suas seqüelas ${ }^{9}$. Entre os adultos, as seqüelas do AVC são consideradas as causas mais comuns de incapacidade ${ }^{10}$. A prevalência de indivíduos com recuperação incompleta tem sido estimada em 460/100.00011, porquanto a incapacidade parcial ou completa está presente entre $24 \%$ e $54 \%{ }^{12}$.

Tendo isso em vista, a QV dessa população tem sido alvo de grande número de pesquisas. Em uma revisão sistemática da literatura realizada por Buck ${ }^{13}$, foram encontrados 15 instrumentos genéricos de QV, mas apenas seis mensuravam multidimensionalmente o construto e apenas sete eram específicos para AVC. Porém, nenhum destes últimos abrangia todos os itens recomendados pela UK National Health Service Research and Development Health Tecchonology Assessment, que incluem confiabilidade, validade, responsividade, adequação, aceitação e modo de administração. Até o presente momento, não há nenhum instrumento para QV específico para AVC adaptado para a população brasileira ${ }^{14}$.

O objetivo deste estudo é identificar os instrumentos genéricos (perfil e utility) e específicos utilizados na avaliação da QV e os seus resultados em sobreviventes de AVC.

\section{MÉTODOS}

Primeiramente, pesquisou-se a terminologia em saúde para QV e AVC, no DeCS (descritores em ciências da saúde) da Biblioteca Virtual em Saúde (BVS). Para o primeiro termo, localizou-se o descritor quality of life e QV, e para o segundo cerebrovascular accident e acidente cerebrovascular, nos idiomas inglês e português. Em seguida, consultaram-se os conceitos dos descritores e seus possíveis sinônimos, incluindo então o termo stroke.

Por meio da BVS, acessou-se a Lilacs (Literatura Latino Americana e do Caribe em Ciências da Saúde), formulário avançado, e foram combinados os descritores em português, sem estabelecer limites. Encontraram-se, então, três publicações. Depois, consultou-se a MedLine (US National Library of Medicine) por meio da PubMed (National Library 
of Medicine and the National Institute of Health). Cruzaramse, então, os descritores em inglês, limitando a pesquisa aos últimos dez anos, com população com 19 anos ou mais e nos idiomas inglês e português. Foram encontradas 262 publicações. Em seguida, os resumos disponíveis foram obtidos no formato abstract plus. A consulta aos bancos de dados virtuais ocorreu no dia 30 de junho de 2007.

Dessa forma, os resumos foram examinados pelos dois autores de maneira independente. Caso o título e o resumo não fossem esclarecedores, buscou-se o artigo na íntegra, para não correr o risco de deixar estudos importantes fora da revisão. Foram considerados relevantes aqueles estudos nos quais a amostra incluía um grupo adequado de participantes e que fosse representativa e bem definida em relação ao diagnóstico clínico; que utilizassem instrumentos padronizados e específicos para o objetivo proposto; cujo período de acompanhamento fosse suficiente e completado adequadamente; e cujos desfechos e exposições correspondessem aos objetivos propostos. O total de 96 estudos foi considerado relevante. Estes foram buscados na literatura para apreciação e inclusão, ou não, neste trabalho.

Para serem incluídas no presente estudo, as publicações deveriam passar pelos seguintes critérios de seleção: conter a descrição da população estudada (idade, sexo, características do AVC e tempo pós-evento), utilizar instrumentos para QV padronizados e validados para o país de origem, estar na língua inglesa ou portuguesa e ter sido publicada entre janeiro de 1996 e junho de 2007. Os estudos exclusivamente qualitativos e aqueles com população menor de 18 anos foram excluídos.

Entre os 96 artigos resgatados da literatura, 31 entraram no presente estudo, de acordo com os critérios de inclusão. Buscaram-se nestes artigos os seus objetivos, o tamanho e as características da amostra, a presença ou não de grupocontrole, o(s) instrumento(s) utilizado(s) na mensuração da QV, bem como a sua qualificação em genérico (perfil ou utility) ou específico, a presença de outros instrumentos empregados e o(s) principal(is) desfecho(s).

As abreviaturas dos instrumentos e de expressões mencionadas no estudo, com seus respectivos significados, encontram-se na Tabela 1.

\section{RESULTADOS}

\section{Instrumentos para qualidade de vida}

Entre os 31 artigos que entraram no presente estudo, 17 (55\%) utilizaram apenas instrumentos genéricos/perfil, seis (19\%) apenas de utility, quatro (13\%) associaram genéricos/ perfil aos utility, três (10\%) abordaram somente específicos e só um (3\%) apresentou a associação entre específico e
Tabela 1. Abreviaturas utilizadas neste estudo.

\begin{tabular}{|c|c|}
\hline AIVD & Atividades instrumentais de vida diária \\
\hline AQOL & Assessement quality of life \\
\hline AVC & Acidente vascular cerebral \\
\hline AVD & Atividades de vida diária \\
\hline $\mathrm{BI}$ & Barthel Index \\
\hline CES-D & Center for Epidemiologic Studies Depression Scale \\
\hline EQ-5D & Euroqol Index \\
\hline EQ-VAS & Euroqol visual analogue scale \\
\hline FAI & Frenchay activities index \\
\hline FIM & Functional independence measure \\
\hline HAD & Hospital anxiety depression scale \\
\hline HUI-2 & Health Utility Index-2 \\
\hline HUI-3 & Health Utility Index-3 \\
\hline MADRS & Montgomery Asberg depression rating scale \\
\hline MBI & Modified Barthel Index \\
\hline MMSE & Mini mental state examination \\
\hline MRS & Modified Rankin Scale \\
\hline NHP & Nottingham Health Profile \\
\hline NIHSS & National Institutes of Health Stroke Scale \\
\hline QLI-stroke version & Ferrans and Powers Quality of Life Index \\
\hline QOL & Quality of life \\
\hline QOL-VAS & Quality of Life Visual Analogue Scale \\
\hline QV & Qualidade de vida \\
\hline QVRS & Qualidade de vida relacionada à saúde \\
\hline RAND-36 & RAND 36 Item Health Survey 1.0 \\
\hline SF-12 & Medical Outcomes Study 12 - Item Short Form Health Survey \\
\hline SF-36 & Medical Outcomes Study 36 - Item Short Form Health Survey \\
\hline SIP & Sickness Impact Profile \\
\hline SIS & Stroke Impact Scale \\
\hline SSS & Scandinavian Stroke Scale \\
\hline тT0 & Time Trade 0ff \\
\hline VAS & Visual Analogue Scale \\
\hline WHOQOL-bref & Brief World Health Organization Quality of Life Assessment Instrument \\
\hline
\end{tabular}

utility. Nenhum fez o uso concomitante de instrumentos específicos e genéricos/perfil.

O instrumento genérico/perfil mais freqüente foi o SF-36, aparecendo em 14 estudos (45,2\%), seguido pelo SF-12, SIP e WHOQOL-bref em três estudos cada (9,7\% cada). Nos trabalhos que abordaram medidas de utility, os mais encontrados foram os EQ-5D e EQ-VAS, em quatro e três publicações $(12,9 \%$ e $9,7 \%)$, respectivamente. Naqueles que fizeram uso de instrumentos específicos predominou o QLI-stroke version, em três trabalhos (9,7\%). No total foram encontrados cinco tipos diferentes de instrumentos genéricos/perfil, nove de utility e dois específicos. A Tabela 2 mostra os instrumentos utilizados na mensuração da QV, sua classificação e os respectivos estudos em que aparecem. 
Tabela 2. Instrumentos utilizados na avaliação da qualidade de vida pós-AVC, sua classificação e os respectivos estudos em que aparecem.

\begin{tabular}{|c|c|c|c|}
\hline Instrumentos & Tipo & Estudo & $\mathrm{n}$ \\
\hline AQOL & Genérico/utility & $\begin{array}{l}\text { Sturm et al. }{ }^{15} \\
\text { Paul et al. } .^{6}\end{array}$ & 2 \\
\hline Direct Scale & Genérico/utility & Samsa ${ }^{10}$ & 1 \\
\hline EQ-5D & Genérico/utility & $\begin{array}{c}\text { Haacke }^{4} \\
\text { Sulch et al. } \\
\text { Xie et al. }{ }^{18} \\
\text { van Exel, Scholte op Reimer; Koopmanschap }{ }^{19}\end{array}$ & 4 \\
\hline EQ-VAS & Genérico/utility & $\begin{array}{l}\text { Sulch et al. }{ }^{17} \\
\text { Xie et al. }{ }^{18} \\
\text { Ahmed et al. } .^{20}\end{array}$ & 3 \\
\hline HUI-2 & Genérico/utility & Haacke $^{4}$ & 1 \\
\hline HUI-3 & Genérico/utility & Haacke $^{4}$ & 1 \\
\hline NHP & Genérico/perfil & Indredavik et al. ${ }^{21}$ & 1 \\
\hline QLI - stroke version & Específico & $\begin{array}{l}\text { Kim et al. } .^{22} \\
\text { Robinson-Smith } \\
\text { Jaracz; Kozubski }\end{array}$ & 3 \\
\hline QOL-VAS & Genérico/utility & Mayo et al. ${ }^{25}$ & 1 \\
\hline SF-12 & Genérico/perfil & $\begin{array}{l}\text { Hackett et al. } .^{26} \\
\text { Xie et al. }{ }^{18} \\
\text { Patel et al. } .^{27}\end{array}$ & 3 \\
\hline SF-36 & Genérico/perfil & $\begin{array}{c}\text { Hopman; Verner } \\
\text { McEwen; Mayo; Wood-Dauphine }{ }^{35} \\
\text { Mayo et al. }{ }^{25} \\
\text { Suenkeler et al. } \\
\text { Widar; Ahlström; } \text { Ek }^{37} \\
\text { Patel et al. }{ }^{27} \\
\text { Ahmed et al. }{ }^{20}\end{array}$ & 14 \\
\hline SIP & Genérico/perfil & $\begin{array}{l}\text { Carod-Artal }{ }^{29} \\
\text { Mackenzie; }{ }^{29 a n 3^{38}} \\
\text { Hochstenbach }\end{array}$ & 3 \\
\hline SIS & Específico & Nichols-Larsen et al. ${ }^{40}$ & 1 \\
\hline тTO & Genérico/utility & Samsa ${ }^{10}$ & 1 \\
\hline VAS & Genérico/utility & $\begin{array}{c}\text { Haacke }^{4} \\
\text { Indredavik et al. }{ }^{21}\end{array}$ & 2 \\
\hline WHOQ0L-bref & Genérico/perfil & $\begin{array}{c}\text { Adams } s^{41} \\
\text { Kwok }^{42} \\
\text { Moon et al. }\end{array}$ & 3 \\
\hline
\end{tabular}

n = freqüência absoluta dos estudos.

\section{Outros instrumentos}

Dos estudos incluídos, 27 (87,1\%) utilizaram outros instrumentos para a mensuração de aspectos específicos que não a QV. Os mais freqüentes foram o BI e o MMSE em 17 e nove estudos, respectivamente, seguidos pelo FAl e MRS em quatro cada, FIM, NIHSS, SSS em três e CES-D, HAD, MADRS e MBI em dois cada. A Tabela 3 mostra estes e os estudos em que aparecem. Além desses, mais 36 tipos diferentes de instrumentos apareceram nos estudos apenas uma vez cada. Ao todo, foram encontrados 47 instrumentos, que mensuravam aspectos, como funcionalidade, ansiedade, depressão, cognição, atenção, destreza, suporte social e emocional, entre outros.

\section{Desfechos}

Os resultados-chave foram agrupados em quatro grupos distintos, de acordo com o método empregado pelos autores.

No primeiro, alocaram-se cinco estudos $(22,7 \%)$ que realizaram apenas uma mensuração em um único momento. No segundo, oito $(36,4 \%)$ apresentaram duas ou mais avaliações, na mesma população, em períodos diferentes. No terceiro, sete $(31,8 \%)$ mostraram resultados referentes a períodos distintos, mas com a presença de grupo-controle. E, no último, foram alocados dois trabaIhos $(9,1 \%)$ que fizeram algum tipo de intervenção com a presença de grupo-controle. 
Tabela 3. Outros instrumentos utilizados nas mensurações pós-AVC, seus objetivos-chave e os estudos onde aparecem.

\begin{tabular}{|c|c|c|c|c|}
\hline Instrumento & Objetivo-chave do instrumento & & & Total \\
\hline Bl & $\begin{array}{l}\text { Avaliar o estado funcional por meio das } \\
\text { atividades de vida diária básicas }\end{array}$ & $\begin{array}{c}\text { Samsa }{ }^{10} \\
\text { Sturm et al. } \\
\text { Carod-Artal }^{129} \\
\text { Haccke } \\
\text { Jönsson et al. }{ }^{31} \\
\text { Sulch et al. } \\
\text { van Exel, Scholte op Reimer; } \\
\text { Koopmanschap }{ }^{19} \\
\text { Aprile et al. } .^{33}\end{array}$ & $\begin{array}{c}\text { McEwen; Mayo; Wood-Dauphine }{ }^{35} \\
\text { Mayo et al.25 } \\
\text { Kauhanen et al.2. } \\
\text { Jaracz; Kozubski } \\
\text { Kwok } \\
\text { Patel et al. }{ }^{27} \\
\text { Moon et al. } .^{23} \\
\text { Ahmed et al. } \\
\text { Indredavik et al. }{ }^{21}\end{array}$ & 17 \\
\hline CES-D & Mensurar a gravidade da sintomatologia da depressão & $\begin{array}{c}\text { Kim et al.22 } \\
\text { Robinson-Smith }\end{array}$ & & 2 \\
\hline FAl & Avaliar o desempenho nas atividades instrumentais de vida diária & $\begin{array}{l}\text { Carod-Artal }\left.\right|^{29} \\
\text { Kim et al. }{ }^{22}\end{array}$ & $\begin{array}{l}\text { Indredavik et al. }{ }^{21} \\
\text { Patel et al. }{ }^{27}\end{array}$ & 4 \\
\hline FIM & Avaliar o estado funcional & $\begin{array}{l}\text { Aprile et al.33 } \\
\text { Kim et al. } .^{22} \\
\text { Robinson-Smith }{ }^{23}\end{array}$ & & 3 \\
\hline HAD & $\begin{array}{l}\text { Rastrear sintomas de ansiedade e depressão sem recorrer } \\
\text { aos sintomas vegetativos }\end{array}$ & $\begin{array}{c}\text { Haacke }^{4} \\
\text { Widar; Ahlström; Ek } \text { Ek }^{37}\end{array}$ & & 2 \\
\hline MADRS & Avaliar os sintomas depressivos & $\begin{array}{l}\text { Naess et al.30 } \\
\text { Suenkeler et al.36 }\end{array}$ & & 2 \\
\hline MBI & $\begin{array}{l}\text { Forma modificada do Bl. Avalia o estado funcional por meio das } \\
\text { atividades de vida diária básicas }\end{array}$ & $\begin{array}{l}\text { Mackenzie; } \text { Chann }^{38} \\
\text { Adams }^{41}\end{array}$ & & 2 \\
\hline MMSE & Rastrear o comprometimento cognitivo & $\begin{array}{c}\text { Haacke }^{4} \\
\text { Jönsson et al. }{ }^{31} \\
\text { Nichols-Larsen et al. }{ }^{40} \\
\text { Kim et al. } \\
\text { Robinson-Smith }\end{array}$ & $\begin{array}{l}\text { Mayo et al.25 } \\
\text { Kauhanen et a. } .^{28} \\
\text { Kwok } \\
\text { Ahmed et al. } .^{20}\end{array}$ & 9 \\
\hline MRS & Avaliar o estado funcional & $\begin{array}{l}\text { Haacke }^{4} \\
\text { Naess et al. }{ }^{30}\end{array}$ & $\begin{array}{l}\text { Sulch et al. }{ }^{17} \\
\text { Aprile et al.33 }\end{array}$ & 4 \\
\hline NIHSS & Avaliar o comprometimento físico & $\begin{array}{l}\text { Sturm et al. }{ }^{15} \\
\text { Paul et al. }{ }^{16} \\
\text { Kwok }^{42}\end{array}$ & & 3 \\
\hline SSS & Avaliar o comprometimento neurológico & $\begin{array}{c}\text { Carod-Artal2 }^{29} \\
\text { Kauhanen et al.28 } \\
\text { Jaracz; Kozubskil }\end{array}$ & & 3 \\
\hline
\end{tabular}

Não obstante a grande variedade de instrumentos (16 para QV e 47 para outras variáveis), os resultados-chave encontrados puderam ser agrupados segundo o método empregado. Na Tabela 4 foram alocados aqueles estudos que objetivaram a obtenção do escore global ou por domínios da QV. Aqueles que visaram a apenas a associação entre esta e outras variáveis não participaram do agrupamento. $\mathrm{O}$ estudo de Jönsson et al..$^{31}$ apresentou dois métodos: comparou a QV da população pós-AVC aos quatro e seis meses, e comparou esta com a de seus cuidadores, que constituíram o grupo-controle. Por isso, foi alocado em dois grupos. Não participaram do agrupamento os estudos que objetivaram unicamente a associação entre QV e outras variáveis e que não apresentaram escore global ou por domínios referentes ao instrumento utilizado.

No primeiro grupo, foram alocados aqueles estudos nos quais a mensuração para QV foi realizada uma única vez, sem presença de grupo-controle. Nas cinco publicações desse agrupamento, houve baixos escores globais ou em pelo menos um domínio da QV.
No segundo, entraram aqueles que mensuraram a QV em um mesmo grupo em dois ou mais momentos distintos. Neste caso, dois desfechos foram encontrados: a melhora ou a piora da QV global ou em algum domínio, em cinco e três estudos, respectivamente. Estes resultados diferem, provavelmente, por causa da variedade de instrumentos e domínios de cada um, às características de cada cultura estudada, ao número de vezes em que ocorreu a mensuração e ao tempo pós-AVC. Este foi o que mais apresentou variações, indo desde a alta hospitalar ${ }^{6}$ até três anos após o evento ${ }^{27}$.

No terceiro, os estudos comparavam os pacientes com outro grupo formado pela população sem AVC. Os trabaIhos de Hackett et al.26, Xie et al.. e Jaracz e Kozubski ${ }^{24}$ encontraram piores escores na população que sofreu AVC, enquanto Anderson et al. ${ }^{32}$ encontraram resultados semelhantes entre os grupos. Este último foi o que mensurou com maior tempo após o evento, que foi de 20 anos. No entanto, houve grande perda de indivíduos neste estudo, que começou com o rastreio de 680 pessoas 
Tabela 4. Principais desfechos quanto à qualidade de vida pós-AVC.

\begin{tabular}{|c|c|c|c|c|c|}
\hline Método & Desfecho & Estudo & $\mathrm{n}$ & Tempo pós-AVC & Total \\
\hline $\begin{array}{l}\text { Uma mensuração em um } \\
\text { único momento }\end{array}$ & $\begin{array}{c}\text { Pobre QV global ou em algum } \\
\text { de seus domínios }\end{array}$ & $\begin{array}{c}\text { Sturm et al. } .^{15} \\
\text { Haacke }^{4} \\
\text { Paul et al. }{ }^{16} \\
\text { Kim et al. } .^{22} \\
\text { Hochstenbach }{ }^{39}\end{array}$ & $\begin{array}{c}225 \\
77 \\
356 \\
55 \\
164\end{array}$ & $\begin{array}{l}7 \text { anos } \\
4 \text { anos } \\
5 \text { anos } \\
\text { Entre } 1 \text { e } 3 \text { anos } \\
9 \text { meses }\end{array}$ & 5 \\
\hline \multirow[t]{2}{*}{$\begin{array}{l}\text { Duas ou mais mensurações } \\
\text { em momentos distintos }\end{array}$} & $\begin{array}{l}\text { Piora da QV global ou em algum de seus } \\
\text { domínios com o passar do tempo }\end{array}$ & $\begin{array}{l}\text { Jönsson et al. }(1)^{31} \\
\text { Suenkeler et al. }{ }^{36} \\
\text { Patel et al.. } \\
\text { Ahmed et al. }(2)^{20} \\
\text { Kwok }{ }^{42}\end{array}$ & $\begin{array}{l}304 \\
144 \\
490 \\
146 \\
303\end{array}$ & $\begin{array}{c}4 \text { e } 6 \text { meses } \\
3,6 \text { e } 12 \text { meses } \\
1 \text { e } 3 \text { anos } \\
1,6 \text { e } 24 \text { semanas } \\
3,6 \text { e } 12 \text { meses }\end{array}$ & 5 \\
\hline & $\begin{array}{l}\text { Melhora da QV global ou em algum de } \\
\text { seus domínios com o passar do tempo }\end{array}$ & $\begin{array}{l}\text { Adams }(3)^{41} \\
\text { Kauhanen et al al } \\
\text { Carod-Artal }^{29}\end{array}$ & $\begin{array}{l}22 \\
85 \\
118\end{array}$ & $\begin{array}{l}\text { Alta hospitalar e após } 1 \text { mês } \\
3 \text { e } 12 \text { meses } \\
\text { Período imediato e após } 1 \text { ano }\end{array}$ & 3 \\
\hline \multirow[t]{3}{*}{$\begin{array}{l}\text { Uma ou mais mensuraçôes } \\
\text { com presença de grupo } \\
\text { controle }\end{array}$} & $\begin{array}{l}\text { Pior QV global ou de algum de seus } \\
\text { domínios quando comparado a um } \\
\text { grupo-controle }\end{array}$ & $\begin{array}{l}\text { Hackett et al. } .^{26} \\
\text { Xie et al. }{ }^{18} \\
\text { Mayo et al. } .^{25} \\
\text { Naess et al. } .^{30} \\
\text { Jaracz; Kozubski }{ }^{24}\end{array}$ & $\begin{array}{c}639 \\
1.040 \\
434 \\
190 \\
72\end{array}$ & $\begin{array}{c}\text { Após } 6 \text { anos } \\
\text { Não institucionalizados } \\
6 \text { meses } \\
\text { Média de } 6 \text { anos } \\
6 \text { meses }\end{array}$ & 5 \\
\hline & $\begin{array}{l}\text { QV do grupo é semelhante ao do } \\
\text { controle }\end{array}$ & Anderson et al..$^{32}$ & 50 & 20 anos & 1 \\
\hline & $\begin{array}{l}\text { QV (domínios físico e social) do grupo é } \\
\text { melhor do que a do controle }\end{array}$ & Jönsson et al. ${ }^{31}$ & 304 & 4 e 6 meses & 1 \\
\hline \multirow[t]{2}{*}{$\begin{array}{l}\text { Comparação entre } \\
\text { intervenções }\end{array}$} & $\begin{array}{l}\text { Melhora da QV no grupo que recebe } \\
\text { cuidado com time multidisciplinar }\end{array}$ & Sulch et al..$^{17}$ & $152(4)$ & 2 semanas e 6 meses & 1 \\
\hline & $\begin{array}{l}\text { Melhora da QV no grupo tratado em uni- } \\
\text { dade específica comparada ao controle }\end{array}$ & Indredavik et al. ${ }^{21}$ & $77(5)$ & Após 5 anos & 1 \\
\hline Total & & & & & $22(6)$ \\
\hline
\end{tabular}

(1) Jönsson = compararam-se os resultados na mesma população e com os de 234 cuidadores; (2) Ahmed = havia 50 cuidadores, mas não houve comparação entre grupos; (3) Adams = 0 estudo pesquisou 22 cuidadores, mas não comparou os resultados com os indivíduos pós-AVC; (4) $152=76$ indivíduos em cuidado com time multidisciplinar e 76 em convencional; (5) $77=45$ em unidade específica e 32 em clínica geral; (6) $22=0$ estudo de Jönsson foi contabilizado duas vezes.

e finalizou com 50. Outra conclusão deste também foi a associação entre o AVC e a baixa expectativa de vida quando comparada aos dados da população geral.

O último agrupamento foi constituído por dois estudos, nos quais houve algum tipo de intervenção com a presença de grupo-controle. No estudo de Sulch et al. ${ }^{17}$, investigaramse as diferenças existentes na QV entre indivíduos sob cuidado convencional e aqueles que recebem tratamento por equipe multidisciplinar. Estes obtiveram os melhores escores. No de Indredavik et al..$^{21}$ compararam-se os resultados entre pacientes atendidos em uma clínica especializada e aqueles em uma de atendimento geral. Os primeiros tiveram maiores escores em todos os domínios, exceto dor, visto que aqueles mais independentes fisicamente também tiveram melhor QV.

Entre aqueles que pesquisaram quais variáveis poderiam influenciar negativamente a QV no indivíduo pós-AVC, foram encontrados: déficit da função física (incluindo os termos funcionalidade, atividades de vida diária, atividades instrumentais de vida diária e destreza), presença de depressão ou de seus sintomas, ser do sexo feminino e o aumento da idade. Essas foram as mais freqüentes e estão em ordem decrescente. A Tabela 5 mostra estas e outras variáveis, bem como suas freqüências.

\section{DISCUSSÃO}

Observando os 31 estudos, nota-se grande variação quanto aos instrumentos utilizados e os resultados obtidos. A maioria utilizou instrumentos genéricos do tipo perfil, o que pode favorecer a comparação entre populações distintas e a avaliação simultânea de várias áreas ou domínios. O mais freqüente foi o SF-36. Este está traduzido para vários idiomas $^{44}$, e é largamente utilizado na população que sofreu $A V C^{45}$. No entanto, efeitos do tipo "chão" e "teto" têm sido demonstrados, segundo Buck'13. Em um estudo de Hobart et al..$^{46}$ sobre os usos e os abusos desse instrumento, cinco dos seus oito domínios têm a validade limitada, como mensuração pós-AVC, levantando-se questões a respeito do seu uso nessa população.

Em uma revisão sistemática da literatura, Tengs, Yu e Luistro $^{3}$ examinaram a variação dos valores atribuídos por indivíduos pós-AVC à QV e ao rigor dos métodos utilizados em estudos que abordaram instrumentos do tipo utility. Foi encontrada enorme variação da QV para um mesmo estado de saúde e considerável diversidade no rigor dos métodos utilizados. A grande heterogeneidade da QV também foi explicada pela variedade de limites inferior e superior das escalas, tornando muitos estudos incomparáveis entre si. 
Tabela 5. Variáveis que se correlacionaram com a QV em indivíduos pós-AVC.

\begin{tabular}{|c|c|c|c|c|}
\hline \multirow[b]{2}{*}{ Variável } & \multicolumn{3}{|c|}{ Correlação com a QV(1) } & \multirow[b]{2}{*}{ Total' } \\
\hline & Positiva & Negativa & Nula & \\
\hline Déficit da função física (3) & 0 & 20 & 2 & 22 \\
\hline Depressão ou seus sintomas & 0 & 12 & 0 & 12 \\
\hline Sexo feminino & 1 & 9 & 2 & 12 \\
\hline Aumento da idade & 0 & 7 & 2 & 9 \\
\hline Características do AVC (2) & 0 & 4 & 1 & 5 \\
\hline Baixo nível educacional & 0 & 2 & 1 & 3 \\
\hline Presença de comorbidades (4) & 0 & 3 & 0 & 3 \\
\hline Ser casado & 2 & 1 & 0 & 3 \\
\hline Qualidade do suporte social & 0 & 2 & 0 & 2 \\
\hline Baixa autoconfiança em realizar auto cuidado & 0 & 1 & 0 & 1 \\
\hline Baixo nível socioeconômico & 0 & 1 & 0 & 1 \\
\hline Comprometimento neurológico & 0 & 1 & 0 & 1 \\
\hline Concordância entre paresia e dominância & 0 & 1 & 0 & 1 \\
\hline Déficit cognitivo & 0 & 1 & 0 & 1 \\
\hline Déficit de atenção & 0 & 1 & 0 & 1 \\
\hline Desemprego & 0 & 1 & 0 & 1 \\
\hline Estar institucionalizado & 0 & 1 & 0 & 1 \\
\hline Fadiga & 0 & 1 & 0 & 1 \\
\hline Incontinência anal & 0 & 1 & 0 & 1 \\
\hline Permanência prolongada no hospital & 0 & 1 & 0 & 1 \\
\hline Praticar poucos exercícios físicos & 0 & 1 & 0 & 1 \\
\hline Presença de dor & 0 & 1 & 0 & 1 \\
\hline Raça/etnia negra & 0 & 1 & 0 & 1 \\
\hline Restrição alimentar & 0 & 1 & 0 & 1 \\
\hline Suporte emocional inadequado & 0 & 1 & 0 & 1 \\
\hline Tipo de dor & 0 & 0 & 1 & 1 \\
\hline Viver sozinho & 0 & 0 & 1 & 1 \\
\hline
\end{tabular}

* Total = referente ao total de estudos que consideraram tais correlações; (1) = Freqüência absoluta dos estudos; (2) = Entende-se por características do AVC, a sua localização, o volume, 0 tipo, a gravidade e a recorrência; (3) = Foram considerados também como função física, a funcionalidade, as atividades de vida diária, as atividades instrumentais de vida diária e a destreza; (4) = Nas comorbidades, excetuou-se ansiedade e depressão, considerando-as separadamente.

Dos estudos abordados, somente quatro fizeram uso de instrumentos específicos. Apesar de a principal característica destes ser a sensibilidade às alterações, nenhum trabalho enfatizou as mudanças da QV diante de alguma intervenção com esse tipo de instrumento.

A escolha do instrumento é item importante, que deve ser associado ao objetivo do estudo e sua disponibilidade no idioma e no contexto cultural da população-alvo7. Além disso, é fundamental saber em que construto ele se fundamenta. Um exemplo é o estudo realizado por Huang, Wu e Frangakis ${ }^{47}$, no qual se questiona se o SF-36 e o WHOQOLbref mensuram o mesmo construto, mesmo sabendo-se que ambos sejam instrumentos genéricos para QV. Foram avaliadas a confiabilidade, a validade de construto e as validades convergentes e divergentes dos instrumentos. Existem diferenças quanto aos objetivos adotados no de- senvolvimento deles. O WHOQOL-bref se fundamenta na concepção de QV da OMS, porquanto os aspectos mensurados abordam a QV global, incluindo os fatores relacionados e não relacionados à saúde. O SF-36 foi desenvolvido para a mensuração dos aspectos da saúde e atividades geralmente afetadas por condições de saúde, avaliando, então, somente a QVRS. Houve também correlações muito fracas entre os seus domínios. Há limitações nesse artigo, como a nacionalidade (Taiwan) e a idade, que ia de 20 a 65 anos. Porém, ele nos faz refletir sobre possível viés ao se compararem os desfechos entre estudos que abordam instrumentos diferentes, mesmo sendo do mesmo tipo e com populações semelhantes.

Levando isso em consideração, é muito difícil fazer comparações entre os estudos aqui incluídos, porém, é possível observar algumas tendências. 
Em 20 de 22 publicações, o déficit da função física foi correlacionado a pior QV. Vários termos foram utilizados para esta variável, como funcionalidade, atividades de vida diária (AVD), atividades instrumentais de vida diária (AIVD) e destreza. No entanto, 17 dos 20 estudos utilizaram o mesmo instrumento desenvolvido por Mahoney e Barthel/8, o Barthel Index (BI). Por este motivo, foi decido agrupá-los, no presente estudo, sob a designação função física. Um projeto desenvolvido no Canadá em 36 centros urbanos e rurais do país investigou os padrões de bem-estar nos idosos que sofreram AVC. Esses tiveram pior sensação de bem-estar, mais comorbidades e maior restrição nas AVD e nas AIVD quando comparados a idosos sem esta patologia ${ }^{49}$.

A presença de depressão ou de seus sintomas também teve associação com pior QV. Dos 12 estudos que abordaram esta variável, todos obtiveram o mesmo desfecho. A prevalência da depressão maior pós-AVC é de 10\% a 34\%, variando conforme o método empregado ${ }^{50}$. Em um estudo multicêntrico na Itália realizado por Toso et al.51, 33,6\% dos 1.064 pacientes obtiveram o diagnóstico de depressão, levando a pensar sobre a importância da mensuração dessa variável nessa população e seu impacto sobre a QV.

Dos nove estudos que avaliaram o impacto da idade sobre a QV, sete mostraram que o aumento da primeira tem impacto negativo na QV. As peculiaridades da população idosa têm levantado questões a respeito dos aspectos psicométricos dos instrumentos utilizados na avaliação desse construto. Pensando nisso, a World Health Organization of Quality of Life Group (WHOQOL Group) está desenvolvendo um instrumento transcultural, o WHOQOL-Old, em 16 centros dos cinco continentes, incluindo o Brasil52.

O sexo feminino também teve influência negativa na QV nos nove dos 12 artigos que abordaram esta variável. Lai et al..$^{53}$ pesquisaram a diferença na recuperação entre os sexos pós-AVC. As mulheres tiveram pior recuperação quanto aos aspectos físicos, apresentavam maior prevalência de sintomas de depressão e eram mais idosas do que os homens, influenciando negativamente em seu estado. No entanto, generalizar este dado, bem como aquele relativo à idade, é controverso, uma vez que essas variáveis são classicamente conhecidas como fatores que necessitam de ajuste nos modelos, seja por interação ou confusão.

Em um primeiro momento, pode parecer inadequado a inclusão neste trabalho dos outros instrumentos utilizados pelos autores que não se referem à QV. No entanto, como este é construto multidimensional, é interessante notar quais áreas estão sendo abordadas em conjunto com a QV, seja em termos de prevalência ou de modificações ao longo do tempo, por meio de instrumentos padronizados. A função física foi a mais avaliada, mostrando alto impacto sobre a QV. No entanto, não podemos valorizar determinado achado com base apenas no número de artigos que o descrevem, uma vez que hipóteses e descobertas originais tendem a aparecer em menos estudos. A Tabela 5 traz diversos outros fatores, como desemprego, baixo nível educacional, incontinência anal e institucionalização.

Seria interessante, no futuro, fazer metanálise com os estudos que utilizaram o mesmo instrumento, como o caso do SF-36, que correspondeu a cerca de $45 \%$ das publicações, identificando os possíveis preditores da QV.

Fica claro, então, a importância do uso dos instrumentos de avaliação da QV, e também do critério de escolha de acordo com os seus objetivos e que os profissionais de saúde possam perceber que esses instrumentos devem ser mais divulgados e aplicados na prática clínica, mostrando a QV como importante ferramenta no rastreio e no acompanhamento dos sobreviventes de AVC ao longo do tempo.

\section{CONCLUSÃO}

A escolha de determinado instrumento deve ser criteriosa, permitindo, assim, a avaliação de aspectos específicos e globais do indivíduo, bem como a comparação entre populações e estudos distintos.

Foi encontrada grande variedade de instrumentos utilizados na mensuração da QV em sobreviventes de AVC. Entre os 16 observados cinco eram genéricos/perfil, nove utility e dois específicos.

Não obstante a grande variação nos métodos e instrumentos empregados, foi possível observar algumas tendências. A pobreza da QV correlacionou-se ao déficit da função física, à presença de depressão ou de seus sintomas, ser do gênero feminino e ser mais idoso. De modo geral, os sujeitos pós-AVC possuíam pior QV do que aqueles que não sofreram o evento.

\section{REFERÊNCIAS}

1. Fleck MPA, Louzada S, Xavier M, Chachamovich E, Vieira G, Santos L, et al. Aplicação da versão em português do instrumento abreviado de avaliação da qualidade de vida "WHOQOL-bref". Rev Saude Publica. 2000;34:178-83.

2. Wyller TB, Holmen J, Laake P, Laake K. Correlates of subjetive well-beging in stroke patients. Stroke. 1998;29:363-7.

3. Tengs T0, Yu M, Luistro E. Health-related quality of life after stroke - a comprehensive review. Stroke. 2001;32:964-72.

4. Haacke C, Althaus A, Spottke A, Siebert U, Back T, Dodel R. Long-term outcome after stroke evaluating health quality of life usuing utility measurements. Stroke. 2006;37:193-8.

5. Gill TM, Feinstein AR. A critical appraisal of the quality of quality-of-life measurements. JAMA. 1994;272:619-26.

6. Campolina AG, Ciconelli RM. Qualidade de vida e medidas de utilidade: parâmetros clínicos para as tomadas de decisão em saúde. Rev Panam Salud Publica. 2006;19:128-36.

7. Ciconelli RM. Medidas de avaliação de qualidade de vida. Rev Bras Reumatol. 2003;43:9-12.

8. Bowling A, Brazier J. Quality of life in social science and medicine. Soc Sci Med. 1995:41:1337-8. 
9. Solomon NA, Glick HA, Russo CJ, Schulman KA. Patient preferences for stroke outcomes. Stroke. 1994;25:1721-5.

10. Samsa GP, Matchar DB. How strong is the relationship between functional status and quality of life among persons with stroke? J Rehab Devel Res. 2004;41:279-82.

11. Bonita R, Solomon N, Broad J. Prevalence of stroke and stroke-related disability: estimates from the Auckland Stroke Studies. Stroke. 1997;27:1898-902.

12. Sacco RL. Risk factors, outcomes and stroke subtypes for ischemic stroke. Neurology. 1997;49 (4 Suppl):39-44.

13. Buck D, Jacoby A, Massey A, Ford G. Evaluation of measures used to assess quality of life after stroke. Stroke. 2000;31:2004-10.

14. Patient-Reported Outcome and Quality of Life Instruments Database. [acesso em 2007 Jun 30]. Disponivel em: http://www.progolid.org.

15. Sturm JW, Donnan GA, Dewey HM, Macdonell RAL, Gilligan AK, Srikanth V, et al. Quality of life after stroke - the North East Melbourne Stroke Incidence Study (NEMESIS). Stroke. 2004:35: 2340-5.

16. Paul SL, Sturm JW, Dewey HM, Donnan GA, Macdonell RAL, Thirft AG. Long-term outcome in the North East Melbourne stroke incidence study - predictors of quality of life at 5 years after stroke. Stroke. 2005;36:2082-6.

17. Sulch D, Melbourn A, Perez I, Kalra L. Integrated care pathways and quality of life on a stroke rehabilitation unit. Stroke. 2002:33:1600-4.

18. Xie J, Wu EQ, Zheng ZJ, Croft JB, Greenlund KJ, Mensah GA, et al. Impact of stroke on healthrelated quality of life in the noninstitutionalized population in the united states. Stroke. 2006; $37: 2567-72$.

19. van Exel NJA, Scholte op Reimer WJM, Koopmanschap MA. Assessment of post-stroke quality of life in cost-effectiveness studies: the usefulness of Barthel Index and the EuroQol-5D. Qual Life Res. 2004:13:427-33.

20. Ahmed S, Mayo NE, Wood-DauphineeS, Hanley JA, Cohen SR. Response Shift influenced estimates of change in health-related quality of life poststroke. J Clin Epidemiol. 2004;56:561-70.

21. Indredavik B, Bakke F, Slórdahl SA, Roksenth R, Hăheim LL. Stroke unit treatment improves long-term quality of life - a randomized controlled trial. Stroke. 1998;29:895-9.

22. Kim P, Warren S, Madill H, Hadley M. Quality of life of stroke survivors. Qual Life Res. 1999:8:293-301.

23. Robinson-Smith G, Johnston MV, Allen J. Self-care self-efficacy, quality of life, and depression after stroke. Arch Phys Med Rehabil. 2000;81:460-4

24. Jaracz K, Kozubski W. Quality of life in stroke patients. Acta Neurol Scand. 2003;107:324-9.

25. Mayo NE, Wood-Dauphinee S, Côté R, Durcan L, Carlton J. Activity, participation, and quality of life 6 months poststroke. Arch Phys Med Rehabil. 2002;83:1035-42.

26. Hackett ML, Duncan JR, Anderson CS, Broad JB, Bonita R. Health-related quality of life among long-term survivors of stroke - results from the Auckland stroke study. 1991-1992. Stroke. 2000:31:440-7.

27. Patel MD, Tilling K, Rudd AG, Wolfe CDA, McKevitt C. Relationships between long-term stroke disability, handicap and health-related quality of life. Age Ageing. 2006;35:273-9.

28. Kauhanen ML, Korpelainen JT, Hiltunen P, Nieminen P, Sotaniemi KA, Myllylä VV. Domains and determinants of quality of life after stroke caused by brain infarction. Arch Phys Med Rehabil. 2000;81:1541-6

29. Carod-Artal J, Egido JA, González JL, Seijas V. Quality of life among stroke survivors evaluated 1 year after stroke: experience of a stroke unit. Stroke. 2000;31:2995-3000.

30. Naess H, Waje-Andreassen U, Thomassen L, Nyland H, Myhr KM. Health-related quality of life among young adults with ischemic stroke on lonf-term follow-up. Stroke. 2006;37:1232-6.
31. Jönsson AC, Lindgren I, Hallström B, Norrving B, Lindgren A. Determinants of quality of life in stroke survivors and their informal caregivers. Stroke. 2005;36:803-8.

32. Anderson CS, Carter KN, Brownlee WJ, Hackett ML, Broad JB, Bonita R. Very long-term outcome after stroke in Auckland, New Zealand. Stroke. 2004;35:1920-4.

33. Aprile I, Piazzini DB, Bertolini C, Caliandro P, Pazzaglia C, Tonali P, et al. Predictive variables on disability and quality of life in stroke outpatients undergoing rehabilitation. Neurol Sci. 2006;27:40-6.

34. Hopman WM, Verner J. Quality of life during and after inpatient stroke rehabilitation. Stroke. 2003;34:801-5.

35. McEwen S, Mayo N, Wood-Dauphinee S. Inferring quality of life from performance-based assessments. Disabil Rehabil. 2000;22:456-63.

36. Suenkeler IH, Nowak M, Mksselwitz B, Kugler C, Schreiber W, Oertel WH, et al. Timecourse of health-related quality of life as determined 3,6 and 12 months after stroke - relationship to neurological deficit, disability and depression. J Neurol. 2002;249:1160-7.

37. Widar $\mathrm{M}$, Ahlström $\mathrm{G}$, Ek AC. Health-related quality of life in persons with long-term pain after a stroke.J Clin Nurs. 2004;13:497-505.

38. Mackenzie AE, Chang AM. Predictors of quality of life following stroke. Disabil Rehabil. 2002;24:259-65

39. Hochstenbach JB, Anderson PG, van Limbeek J, Mulder TT. Is there a relation between neuropsychologic variables and quality of life after stroke? Arch Phys Med Rehabil. 2001;82:1360-6.

40. Nichols-Larsen DS, Clark PC, Zeringue A, Greenspan A, Blanton S. Factors influencing stroke survivors'quality of life during subacute recovery. Stroke. 2005;36:1480-4.

41. Adams C. Quality of life for caregivers and stroke survivors in the immediate discharge period. Appl Nurs Res. 2003;16:126-30.

42. Kwok T, Lo RS, Wong E, Wai-Kwong T, Mok V, Kai-Sing W. Quality of life of stroke survivors: a 1-year follow-up study. Arch Phys Med Rehabil. 2006;87:1177-82.

43. Moon YS, Kim SJ, Kim HC, Won MH, Kim DH. Correlates of quality of life after stroke. J Neurol Sci. 2004;224:37-41.

44. Ciconelli RM, Ferraz MB, Santos W, Meinão I, Quaresma MR. Tradução para a língua portuguesa e validação do questionário genérico de avaliação de qualidade de vida SF-36 (Brasil SF-36). Rev Bras Reumatol. 1999:39:143-50.

45. Haan RJ. Measuring quality of life after stroke using the SF-36. Stroke. 2002;33:1176-7.

46. Hobart JC, Williams LS, Moran K, Thompson AJ. Quality of life measurement after stroke uses and abuses of the SF-36. Stroke. 2002;33:1348-56.

47. Huang IC, Wu AW, Frangakis C. Do the SF-36 and WHOQOL-BREF measure the same constructs? Evidence from the Taiwan population. Qual Life Res. 2006;15:15-24.

48. Mahoney Fl, Barthel DW. Functional evaluation: the Barthel Index. Maryland State Med J. 1965;14:61-5.

49. Clarke P, Marshall V, Black SE, Colantonio A. Well-being after stroke in Canadian seniors finding from tne Canadian study of health and aging. Stroke. 2002;33:1016-21.

50. Terroni LMN, Leite CC, Tinone G, Fráguas Jr R. Depressão pós-AVC: fatores de risco e terapêutica antidepressiva. Rev Assoc Med Bras. 2003:49:450-9.

51. Toso V, Gandolfo C, Paolucci S, Provinciali L, Torta R, Grassivaro N. Post-stroke depression: research methotology of a large multicentre observacional study (DESTRO). Neurol Sci. 2004;25:138-44

52. Fleck MPA, Chachamovich E, Trentini CM. WHOQOL-OLD project: method and focus group results in Brazil. Rev Saude Publica. 2003;33:1-11.

53. Lai S-M, Duncan PW, Dew P, Keighley J. Sex differences in stroke recovery. Prev Chronic Dis. 2005;2:1-11. 